Pacific Journal of Mathematics

ON CONFORMAL MAPPING OF NEARLY CIRCULAR 


\section{ON CONFORMAL MAPPING OF NEARLY CIRCULAR REGIONS}

\section{DIETER GAIER}

Introduction. A Jordan curve $C$ in the $w$-plane, starshaped with respect to $w=0$ and represented in polar coordinates by $\rho(\theta) e^{i \theta}$, will be said to satisfy an $\varepsilon$-condition $(\varepsilon \geqq 0)$ if

$$
\begin{aligned}
& \text { (i) } \rho(\theta) \text { is absolutely continuous in }\langle-\pi,+\pi\rangle \\
& \text { (ii) }\left|\frac{\rho^{\prime}}{\rho}(\theta)\right| \leqq \varepsilon \text { for almost all } \theta \text { in }\langle-\pi,+\pi\rangle \text {. }
\end{aligned}
$$

Sometimes the condition

$$
1 \leqq \rho(\theta) \leqq 1+\varepsilon \text { for all } \theta \text { in }\langle-\pi,+\pi\rangle
$$

will be added.

Let $w=f(z)$ be the conformal mapping of $|z|<1$ to the interior of $C$ such that $f(0)=0, f^{\prime}(0)>0$. Then one can ask: How "close" is $f(z)$ to the identity mapping $z$ ? This question has been studied by many authors, notably Marchenko [3] and, more recently, by Warschawski [9-14] and Specht [7]. For example, Marchenko stated:

THEOREM A. If $C$ satisfies an e-condition and also (0.2), then

$$
|f(z)-z| \leqq K \cdot \varepsilon
$$

for a universal constant $K$.

Furthermore, estimates for $M_{p}\lfloor f(z)-z]$ and $M_{p}\left[f^{\prime}(z)-1\right]$ have been given [9] where we write, for example,

$$
\begin{aligned}
&\|f(z)-z\|_{p} \equiv M_{p}[f(z)-z]=\left\{\frac{1}{2 \pi} \int_{0}^{2 \pi}\left|f\left(r e^{i \varphi}\right)-r e^{i \varphi}\right|_{p_{d \varphi}}\right\}^{1 / p} \\
&(p>0 ;|z|=r<1) .
\end{aligned}
$$

In this connection, the theorem of M. Riesz [6] on conjugate harmonie functions is of importance.

Theorem B. Let $f(z)=u(z)+i v(z)$ be regular in $|z|<1$ and $v(0)=0$, so that $v(z)$ is a "normed conjugate" of $u(z)$. Then for every $p>1$

$$
M_{p}[v(z)] \leqq A_{p} M_{p}[u(z)] \quad(|z|=r<1),
$$

Received February 23, 1961. The preparation of this paper was supported in part by the Office of Naval Research. 
where $A_{p}$ is a constant that depends on $p$ only; one can take $A_{2}=1$, $A_{p} \leqq 2 p(p \geqq 2)$ and $A_{p^{\prime}}=A_{p}$ for $p^{-1}+p^{-1}=1$. If the right-hand side of $(0.4)$ is bounded in $0 \leqq r<1$, then $f\left(r e^{i \varphi}\right)$ has radial boundary values of class $L_{p}$ almost everywhere and (0.4) holds for $r=1$.

In this paper we would like to make a few remarks about Marchenko's theorem and about estimates for $M_{p}\left[f^{\prime}(z)-1\right]$. First, we give a new proof of Theorem A which we hope is slightly simpler than Specht's [7] while giving only a slightly larger constant $K$. Next we ask whether we could replace the condition (0.1.ii) by the assumption of convexity of $C$ and still get (0.3). A counter example is constructed in I.2. Then Specht's method of proof is used to give a localized version of Theorem $\mathrm{A}$, in which the $\varepsilon$-condition is fulfilled only for a part of $C$.

In the second part of the paper we obtain new estimates for $M_{p}\left[f^{\prime}(z)-1\right]$. Their source is a sharp and best possible estimate for $\int_{0}^{2 \pi}\left[\theta^{\prime}(\varphi)\right]^{ \pm p} d \varphi$ where $\theta(\varphi)=\arg f\left(e^{i \varphi}\right)$. It avoids the restriction $\varepsilon<1$ of Warschawski [9] and gives all values of $p$ for which $M_{p}\left[\theta^{\prime}(\varphi)\right]<\infty$ or $M_{p}\left[f^{\prime}(z)\right]$ remains bounded for all $r=|z|<1$.

\section{PART I}

I.1. New proof of Marchenko's theorem. While Specht's approach to Theorem A depends on a suitable integral representation of $\theta(\varphi)-\varphi$ ([7], p. 187), and Warschawski's on an estimate of $M_{2}\left[f^{\prime}\left(e^{i \varphi}\right)-1\right]$ ([9], p. 566), our proof will depend on a sharp estimate of $M_{2}\left[\theta^{\prime}(\varphi)-1\right]$. We shall prove:

THEOREM 1. If the Jordan curve $C$ satisfies an e-condition and also (0.2) for some $\varepsilon \geqq 0$, then $|f(z)-z| \leqq K(\varepsilon) \cdot \varepsilon$ in $|z| \leqq 1$, where $K(\varepsilon) \leqq 3.7$ for all $\varepsilon \geqq 0$ and $\lim _{\epsilon \rightarrow 0} K(\varepsilon)=\sqrt{1+\pi^{2} / 3} \sim 2.1$.

Specht's proof yields another function $\bar{K}(\varepsilon)$ with $\bar{K}(\varepsilon) \leqq 3.3$ and $\lim _{\epsilon \rightarrow 0} \bar{K}(\varepsilon)=\sqrt{1+(2 \log 2)^{2}} \sim 1.7$. The best possible bounds are not known.

In order to prove the theorem, we need the following

LEMma. Let $F(x)$ be absolutely continuous in $\langle 0,2 \pi\rangle$, periodic with $2 \pi$ and $\int_{0}^{2 \pi} F(x) d x=0$, and assume $F^{\prime}(x) \in L_{2}(0,2 \pi)$. Then for all $x$ in $\langle 0,2 \pi\rangle$

$$
|F(x)| \leqq \frac{\pi}{\sqrt{3}}\left\|F^{\prime}(x)\right\|_{2}
$$


This lemma is also used in Friberg's thesis ([2], p. $14 \mathrm{ff}$ ). The constant $\frac{\pi}{\sqrt{3}}$ cannot be improved as $F(x)=\frac{x^{2}}{4}-\frac{\pi}{2} x+\frac{\pi^{2}}{6}(0 \leqq x \leqq 2 \pi)$ shows.

Proof. It suffices to estimate $F(0)$. For that we expand $F(x)$ in its Fourier series $F(x)=\sum_{n=1}^{\infty}\left(a_{n} \cos n x+b_{n} \sin n x\right)$ and get

$$
|F(0)|=\left|\sum_{1}^{\infty} a_{n}\right| \leqq \sum_{1}^{\infty}\left|a_{n}\right| \cdot n \frac{1}{n} \leqq\left[\sum_{1}^{\infty} n^{2} a_{n}^{2}\right]^{1 / 2}\left[\sum_{1}^{\infty} n^{-2}\right]^{1 / 2} .
$$

The first factor is at most $\left[\sum_{1}^{\infty} n^{2}\left(a_{n}^{3}+b_{n}^{2}\right)\right]^{1 / 2}=\sqrt{2}\left\|F^{\prime}(x)\right\|_{2}$, by Parseval's equality, the second is $\pi / \sqrt{6}$.

Proof of the theorem. Putting $f\left(e^{i \varphi}\right)=\rho(\theta) e^{i \theta}, \theta=\theta(\varphi)$, we first estimate $|\theta(\varphi)-\varphi|$ if $\varepsilon$ is assumed to be $<1$. By the lemma, it is sufficient to estimate $\left\|\theta^{\prime}(\varphi)-1\right\|_{2}$. To do this, we note that $\log (f(z) / z)=$ $u(z)+i v(z)$ is regular in $|z|<1$, continuous in $|z| \leqq 1$, and $v(0)=0$ since $f^{\prime}(0)>0$, so that $v$ is a normed conjugate of $u: v=K[u]$. On $|z|=1$ this gives

$$
\theta(\varphi)-\varphi=K[\log \rho(\theta(\varphi))], \theta(\varphi+h)-(\varphi+h)=K[\log \rho(\theta(\varphi+h))]
$$

and hence

$$
\frac{\theta(\varphi+h)-\theta(\varphi)}{h}-1=K\left[\frac{\log \rho(\theta(\varphi+h))-\log \rho(\theta(\varphi))}{h}\right] .
$$

By (0.1), for all $\varphi$ and $h>0$

$$
|\log \rho(\theta(\varphi+h))-\log \rho(\theta(\varphi))|=\left|\int_{\theta(\varphi)}^{\theta(\varphi+h)} \frac{\rho^{\prime}}{\rho}(t) d t\right| \leqq \varepsilon|\theta(\varphi+h)-\theta(\varphi)|,
$$

and therefore

$$
\left\|\frac{\theta(\varphi+h)-\theta(\varphi)}{h}-1\right\|_{2}=\|[\quad]\|_{2} \leqq \varepsilon\left\|\frac{\theta(\varphi+h)-\theta(\varphi)}{h}\right\|_{2} .
$$

Now we claim that

$$
\left\|\frac{\theta(\varphi+h)-\theta(\varphi)}{h}-1\right\|_{2}^{2}=\left\|\frac{\theta(\varphi+h)-\theta(\varphi)}{h}\right\|_{2}^{2}-1 \text {. }
$$

To show this, we write the left-hand side as

$$
\begin{array}{r}
\frac{1}{2 \pi} \int_{0}^{2 \pi}\left[\frac{\theta(\varphi+h)-\theta(\varphi)}{h}-1\right]^{2} d \varphi=\frac{1}{2 \pi} \int_{0}^{2 \pi}\left[\frac{\theta(\varphi+h)-\theta(\varphi)}{h}\right]^{2} d \varphi+1 \\
-2 \frac{1}{2 \pi h} \int_{0}^{2 \pi}\{[\theta(\varphi+h)-(\varphi+h)]-[\theta(\varphi)-\varphi]+h\} d \varphi .
\end{array}
$$


Since $\theta(\varphi)-\varphi$ is periodic with $2 \pi$, the last term is -2 , and (1.4) follows. Together with (1.3) we get $\|[\theta(\varphi+h)-\theta(\varphi)] / h-1\|_{2}^{2} \leqq \varepsilon^{2} /\left(1-\varepsilon^{2}\right)$ for all $h>0$. But since $C$ is rectifiable, $\theta(\varphi)$ is absolutely continuous [5] and hence $\theta^{\prime}(\varphi)$ exists almost everywhere, and Fatou's lemma yields now for $h \rightarrow 0$

$$
\left\|\theta^{\prime}(\varphi)-1\right\|_{2} \leqq \frac{\varepsilon}{\sqrt{1-\varepsilon^{2}}} .
$$

This, incidentally, is a best possible estimate; see Theorem 6 .

Now we apply our lemma to $F(\varphi)=\theta(\varphi)-\varphi$, the condition $\int_{0}^{2 \pi} F(\varphi) d \varphi=0$ following from (1.2), and we get for all $\varphi$

$$
|\theta(\varphi)-\varphi| \leqq \frac{\pi}{\sqrt{3}} \frac{\varepsilon}{\sqrt{1-\varepsilon^{2}}} \cdot{ }^{2}
$$

From this we obtain an estimate of $|f(z)-z|$. An elementary consideration gives

$$
|f(z)-z|^{2} \leqq \varepsilon^{2}+(1+\varepsilon)[\theta(\varphi)-\varphi]^{2} \text { on }|z|=1 ;
$$

note that $1 \leqq\left|f\left(e^{i \varphi}\right)\right| \leqq 1+\varepsilon$. Together with (1.6) we obtain

$$
|f(z)-z| \leqq \varepsilon\left\{1+\frac{\pi^{2}}{3(1-\varepsilon)}\right\}^{1 / 2}
$$

for $|z|=1$ and hence, by the maximum principle, for $|z| \leqq 1$; this is valid whenever $\varepsilon<1$. For all $\varepsilon \leqq 20 / 27$ the factor of $\varepsilon$ is $\leqq 3.7$; for $\varepsilon>20 / 27$ we have

$$
|f(z)-z| \leqq 1+\varepsilon+1=2+\varepsilon<\frac{54}{20} \varepsilon+\varepsilon=3.7 \varepsilon .
$$

This proves $K(\varepsilon) \leqq 3.7$ for all $\varepsilon \geqq 0$, and (1.8) gives $\lim _{\epsilon \rightarrow 0} K(\varepsilon)=\sqrt{1+\pi^{2} / 3}$.

Specht ([7], p. 188) obtains $|\theta(\varphi)-\varphi| \leqq \varepsilon(2 \log 2+\varepsilon)$. Combining this for $\varepsilon \leqq 0.9$ with $|f(z)-z| \leqq \varepsilon+|\theta(\varphi)-\varphi|(|z|=1)$ and taking $|f(z)-z| \leqq 2+\varepsilon$ for $\varepsilon>0.9$, one obtains $\bar{K}(\varepsilon) \leqq 3.3$ for all $\varepsilon>0$; for $\varepsilon \rightarrow 0$ use (1.7).

I.2. Convex regions. Our next problem is to decide whether Marchenko's theorem remains valid if the condition $\left|\rho^{\prime}\right| \rho \mid \leqq \varepsilon$ is replaced by the convexity of $C$. To study a suitable counter example, it will be convenient to use the following localization theorem.

${ }_{1}$ This also follows directly from $\left\|\theta^{\prime}\right\|_{2} \leqq\left(1-\varepsilon^{2}\right)^{-1 / 2}\left([\mathbf{8}]\right.$, p. 26) and $\left\|\theta^{\prime}-1\right\|_{2}^{2}=\left\|\theta^{\prime}\right\|_{2}^{2}-1$, but we wanted to give an independent proof of (1.5).

2 The application of Warschawski's inequality ([8], p. 18) would have given a slightly larger bound for $K$ in Theorem 1 , 
Theorem 2. Let $C: \rho(\theta) e^{i \theta}$ be a Jordan curve, starshaped with respect to $w=0$ and contained in $1 \leqq|w| \leqq 1+\varepsilon$, and let $w=f(z)$ with $f(0)=0, f^{\prime}(0)>0$ map $|z|<1$ conformally to the interior of $C$; put $\theta(\varphi)=\arg f\left(e^{i \varphi}\right)$.

Then to every $\delta, 0<\delta<\pi$, corresponds a constant $D=D(\delta)$ such that

$$
\left|[\theta(\varphi)-\varphi]-\frac{1}{2 \pi} \int_{\varphi-\delta}^{\varphi+\delta} \log \rho(\theta(t)) \operatorname{ctg} \frac{\varphi-t}{2} d t\right| \leqq D \cdot \varepsilon
$$

for all $\varphi$, the integral being a Cauchy principal value.

Proof. Since $\theta(\varphi)-\varphi$ is a normed conjugate of $\log \rho(\theta(\varphi)$ ) (see (1.2)), we have

$$
\theta(\varphi)-\varphi=\frac{1}{2 \pi} \int_{\varphi \rightarrow \pi}^{\varphi+\pi} \log \rho(\theta(t)) \operatorname{ctg} \frac{\varphi-t}{2} d t=\frac{1}{2 \pi} \int_{\varphi-\delta}^{\varphi+\delta}+\frac{1}{2 \pi}\left[\int_{\varphi-\pi}^{\varphi-\delta}+\int_{\varphi+\delta}^{\varphi+\pi}\right] .
$$

In the last term $|\operatorname{ctg}[(\varphi-t) / 2]|$ is bounded by $\operatorname{ctg}[\delta / 2]$ while $0 \leqq \log$ $\rho(\theta(t)) \leqq \varepsilon$. This proves (1.9) with $D(\delta)=\operatorname{ctg}[\delta / 2]$.

Furthermore, we shall use another theorem of Marchenko ([3], p. 289) which, in the generalization by Warschawski ([10], p. 343), reads as follows. Let $R$ be a simply connected region containing $w=0$ whose boundary is contained in $1 \leqq|w| \leqq 1+\varepsilon$. Let $\lambda$ be such that any two points in $R$ with distance $<\varepsilon$ may be connected in $R$ by an arc of diameter $<\lambda$. If $f(z)$ is the normalized mapping of $|z|<1$ to $R$, then

$$
|f(z)-z| \leqq M \varepsilon|\log \varepsilon|+M_{1} \lambda
$$

for two absolute constants $M$ and $M_{1}$. Ferrand ([1], p. 133) states without proof that one can take $M=1 / \pi$ as the best possible constant; note that in her paper the boundary is assumed to be in $1-\varepsilon \leqq|w| \leqq 1+\varepsilon$.-Obviously $\lambda \leqq 3 \varepsilon$ if $R$ is starshaped with respect to $w=0$.

Now we shall study the following family of conformal maps. Let the Jordan curve $C=C(\varepsilon)(0<\varepsilon<1 / 2)$ be defined as follows:

$$
\begin{array}{rr}
|w|=1 \quad \text { if }-\pi \leqq \arg w \leqq 0, & \text { if } 0<\theta_{2} \leqq \arg w \leqq \frac{\pi}{2}+\kappa, \text { where } 0<\kappa<\frac{\pi}{2} \text { and } \\
|w|=1+\varepsilon & \sin \kappa=1 /(1+\varepsilon),
\end{array}
$$

and where these two circular arcs are connected by straight line segments. The angle $\theta_{2}$ will also depend on $\varepsilon$ and is subject to 


$$
\theta_{2} \rightarrow 0 \text { and } \frac{\theta_{2}}{\varepsilon|\log \varepsilon|} \rightarrow+\infty \quad(\varepsilon \rightarrow 0)
$$

Let $w=f(z)$ map $|z|<1$ to the interior of $C$ with $f(0)=0, f(0)>0$ and let

$$
f\left(e^{i \varphi_{1}}\right)=1=e^{i \theta_{1}}, f\left(e^{i \varphi_{2}}\right)=(1+\varepsilon) e^{i \theta_{2}} .
$$

By (1.10) we have for all $\varphi$ and $\varepsilon$

$$
|\theta(\varphi)-\varphi| \leqq M \varepsilon|\log \varepsilon|+O(\varepsilon),
$$

in particular $\varphi_{1} \rightarrow 0, \varphi_{2} \rightarrow 0(\varepsilon \rightarrow 0)$. We therefore get from Theorem 2

$$
\begin{aligned}
\theta\left(\varphi_{1}\right)-\varphi_{1} & =\frac{1}{2 \pi} \int_{-\pi / 2}^{+\pi / 2} \log \rho(\theta(t)) \operatorname{ctg} \frac{\varphi_{1}-t}{2} d t+O(\varepsilon) \\
\left|\theta\left(\varphi_{1}\right)-\varphi_{1}\right| & =\frac{1}{2 \pi} \int_{\varphi_{1}}^{+\pi / 2} \log \rho(\theta(t)) \operatorname{ctg} \frac{t-\varphi_{1}}{2} d t+O(\varepsilon)>\frac{1}{2 \pi} \int_{\varphi_{2}}^{+\pi / 2}+O(\varepsilon) ;
\end{aligned}
$$

note that $\rho(\theta(t))=1$ for $t$ in $\left\langle\pi / 2, \varphi_{1}\right\rangle$. The last integral is equal to

$$
\log (1+\varepsilon) \int_{\varphi_{2}}^{+\pi / 2} \operatorname{ctg} \frac{t-\varphi_{1}}{2} d t=2\left|\log \left(\varphi_{2}-\varphi_{1}\right)\right| \varepsilon+O(\varepsilon)
$$

Here we have by (1.11) and (1.12)

$$
\varphi_{2}-\varphi_{1}=\theta_{2}-\theta_{1}+O(\varepsilon|\log \varepsilon|)=\left(\theta_{2}-\theta_{1}\right)(1+o(1))=\theta_{2}(1+o(1)),
$$

so that altogether we obtain

$$
\left|\theta\left(\varphi_{1}\right)-\varphi_{1}\right|>\frac{\left|\log \theta_{2}\right|}{\pi} \varepsilon+O(\varepsilon)
$$

Before we specialize (1.13), we remark that for the regions considered here

$$
|f(z)-z|=|\theta(\varphi)-\varphi|+O(\varepsilon) \quad\left(z=e^{i \varphi}\right) .
$$

We have namely on $|z|=1$

$$
2 \sin \frac{\theta(\varphi)-\varphi}{2} \leqq|f(z)-z| \leqq 2 \sin \frac{\theta(\varphi)-\varphi}{2}+(|f(z)|-1) .
$$

By (1.12), $|\theta(\varphi)-\varphi|=O(\varepsilon|\log \varepsilon|)$ and (1.14) follows.

We now make two special choices of $\theta_{2}=\theta_{2}(\varepsilon)$, always subject to (1.11). For our first choice $\theta_{2}(\varepsilon)=\varepsilon|\log \varepsilon|^{2}$ we obtain from (1.13) and (1.14) 


$$
|f(z)-z| \geqq \frac{\varepsilon|\log \varepsilon|}{\pi}(1+o(1)) \quad\left(z=e^{i \varphi_{1}} ; \varepsilon \rightarrow 0\right) .
$$

Thus we proved that the best constant $M$ in (1.10) must satisfy $M \geqq 1 / \pi$, in agreement with Ferrand.

Next we choose $\theta_{2}$ such that $1=(1+\varepsilon) \cos \theta_{2}$, which makes $C(\varepsilon)$ convex. If we insert $\theta_{2}=\sqrt{2 \varepsilon}+O(\varepsilon)$ in (1.13), we obtain

$$
|f(z)-z| \geqq \frac{\varepsilon|\log \varepsilon|}{2 \pi}(1+o(1)) \quad\left(z=e^{i \varphi_{1}} ; \varepsilon \rightarrow 0\right) .
$$

Theorem 3. If $C(\varepsilon)$ is the family of convex curves defined by cos $\theta_{2}(\varepsilon)=[1 / 1+\varepsilon]$, we have

$$
\max _{|z|=1}|f(z)-z| \geqq \frac{\varepsilon|\log \varepsilon|}{2 \pi}(1+o(1)) \quad(\varepsilon \rightarrow 0) .
$$

In particular, Theorem $A$ does not hold if the condition $\left|\rho^{\prime}(\theta)\right| \rho(\theta) \mid \leqq \varepsilon$ is replaced by the convexity of $C$.

I.3. Localization of the theorem of Marchenko. In I. 1 we have seen that Theorem A can be proved with a quite satisfactory constant $K$ by a "global" method, a method involving means rather than the function itself. Nevertheless, Specht's proof of Theorem A, directly aiming at $|\theta(\varphi)-\phi|$, has besides giving a slightly better constant the advantage of being useful to obtain a localization of Theorem A, where $\left|\left[\rho^{\prime} \mid \rho\right]\right| \leqq \varepsilon$ is known only for a part of $C$.

We begin with the following localization of Specht's representation theorem ([7], p. 187).

Theorem 4. Let $C: \rho(\theta) e^{i \theta}$ be a Jordan curve, starshaped with respect to $w=0$ which satisfies :

(i) $1 \leqq \rho(\theta) \leqq 1+\varepsilon$ for all $\theta$ and some $\varepsilon \geqq 0$;

(ii) $\rho(\theta)$ has bounded difference quotients for $\theta$ in $\langle a, b\rangle$.

Then to every $\delta>0$ corresponds an $\varepsilon_{0}=\varepsilon_{0}(\delta)>0$ and a constant $N(\delta)$ such that for $\varepsilon<\varepsilon_{0}$ we have

$$
\left|[\theta(\varphi)-\varphi]-\frac{1}{\pi} \int_{a}^{b} \log \right| \sin \frac{t(\theta)-\varphi}{2}\left|\frac{\rho^{\prime}(\theta)}{\rho(\theta)} d \theta\right| \leqq N(\delta) \cdot \varepsilon,
$$

for all $\varphi$ in $\langle a+\delta, b-\delta\rangle$ for which $\theta^{\prime}(\varphi)$ and $\rho^{\prime}(\theta(\varphi))$ exist and $\theta^{\prime}(\dot{\varphi}) \neq 0$, i.e. for almost all $\varphi$ in $\langle a+\delta, b-\delta\rangle$.

Here $t=t(\theta)$ is the inverse function of $\theta(t)$, and the integral exists as a Lebesgue integral. 
Proof. For our fixed $\delta>0$, choose $\varepsilon_{0}(\delta)$ such that $\alpha=\theta^{-1}(a)$ and $\beta=\theta^{-1}(b)$ satisfy $|\alpha-a|<\delta / 2,|\beta-b|<\delta / 2$; this is asserted by (1.10) or (1.12) as soon as $\varepsilon<\varepsilon_{0}$. Then we can write

$$
\begin{aligned}
\theta(\varphi)-\varphi & =\frac{1}{2 \pi} \int_{\varphi-\pi}^{\varphi+\pi}[\log \rho(\theta(t))-\log \rho(\theta(\varphi))] \operatorname{ctg} \frac{\varphi-t}{2} d t \\
& =\frac{1}{2 \pi} \int_{\alpha}^{\beta}\left[1 \operatorname{ctg} \frac{\varphi-t}{2} d t+O(\varepsilon) ;\right.
\end{aligned}
$$

compare Theorem 2. Now one applies partial integration to the integral as in Specht's proof, and (1.15) follows.

Now we can prove the following localization of Theorem A.

THeOREM 5. Let $C: \rho(\theta) e^{i \theta}$ be a rectifiable Jordan curve, starshaped with respect to $w=0$ which satisfies

(i) $1 \leqq \rho(\theta) \leqq 1+\varepsilon$ for all $\theta$ and some $\varepsilon \geqq 0$,

(ii) $|\rho(\theta+\tau)-\rho(\theta)| \leqq \rho(\theta)|\tau| \varepsilon$ for all $\theta$ in $\langle a, b\rangle$ and all real $\tau$. Then to every $\delta>0$ corresponds a constant $K_{1}(\delta)$ such that

$$
|f(z)-z| \leqq K_{1}(\delta) \cdot \varepsilon \text { for } z=e^{i \varphi}, \varphi \text { in }\langle a+\delta, b-\delta\rangle \text {. }
$$

Proof. It suffices to prove this for small $\varepsilon$. Condition (ii) implies that we can estimate the integral term in (1.15) by

$$
\begin{aligned}
\varepsilon\left|\frac{1}{\pi} \int_{a}^{b} \log \right| \sin \frac{t(\theta)-\varphi}{2}|d \theta| & \leqq-\varepsilon \frac{1}{\pi} \int_{\varphi-\pi}^{\varphi+\pi} \log \left|\sin \frac{t-\varphi}{2}\right| \theta^{\prime}(t) d t \\
& \leqq \varepsilon(2 \log 2+\varepsilon)
\end{aligned}
$$

(see [7], p. 188). Hence $|\theta(\varphi)-\varphi| \leqq K_{2}(\delta) \cdot \varepsilon$ for almost all $\varphi$ in $\langle a+\delta, b-\delta\rangle$. By continuity, this holds for all $\varphi$ in $\langle a+\delta, b-\delta\rangle$, and (1.16) follows.

REMARK. By a simple approximation argument it is seen that the rectifiability of $C$, needed for the last inequality in (1.17), is not necessary for the validity of Theorem 5 .

\section{PART II}

II. 1. Sharp estimates for the means of $\theta^{\prime}(\varphi)$. Our aim is now to give an estimate for $M_{p}\left[f^{\prime}(\mathrm{z})-1\right]$ if $C$ satisfies an $\varepsilon$-condition. As a first step we prove the following

TheOREM 6. Let $C: \rho(\theta) e^{i \theta}$ be a Jordan curve, starshaped with respect to $w=0$, which satisfies an $\varepsilon$-condition for some $\varepsilon \geqq 0$, and 
let $w=f(z)$ with $f(0)=0$ map $|z|<1$ conformally to the interior of C. Then $\theta(\varphi)=\arg f\left(e^{i \varphi}\right)$ satisfies

$$
\int_{0}^{2 \pi}\left[\theta^{\prime}(\varphi)\right]^{ \pm p} d \rho<\infty \text { if } 0 \leqq p<\frac{\pi}{2 \operatorname{arctg} \varepsilon} .
$$

More precisely, we have

$$
\begin{aligned}
\frac{1}{2 \pi} \int_{0}^{2 \pi}\left[\theta^{\prime}(\varphi)\right]^{p} d \varphi & \leqq \frac{(\cos \operatorname{arctg} \varepsilon)^{p}}{\cos (p \operatorname{arctg} \varepsilon)} \text { if } 1 \leqq p<\frac{\pi}{2 \operatorname{arctg} \varepsilon} \\
& \leqq 1 \quad \text { if } 0 \leqq p \leqq 1
\end{aligned}
$$

and

$$
\begin{aligned}
\frac{1}{2 \pi} \int_{0}^{2 \pi}\left|\theta^{\prime}(\varphi)\right|^{-p} d \varphi \leqq \frac{1}{(\cos \operatorname{arctg} \varepsilon)^{p} \cos (p \operatorname{arctg} \varepsilon)} \\
\text { if } 0 \leqq p<\frac{\pi}{2 \operatorname{arctg} \varepsilon} .
\end{aligned}
$$

Moreover, the bounds in (2.2) and (2.3), as well as the upper bound for $p$ in (2.1), are best possible.

REMARKs. a. It easily follows from F. Riesz's theorem ([5], p. 95), that not only $\theta=\theta(\varphi)$ but also its inverse $\varphi=\varphi(\theta)$ is an absolutely continuous and monotonically increasing function, whenever $C$ satisfies an $\varepsilon$-condition for some $\varepsilon \geqq 0$. The substitution $\varphi=\varphi(\theta)$ in (2.1) is therefore permissible ${ }^{3}$ and gives

$$
\frac{1}{2 \pi} \int_{0}^{2 \pi}\left[\theta^{\prime}(\varphi)\right]^{ \pm p} d \varphi=\frac{1}{2 \pi} \int_{0}^{2 \pi}\left[\varphi^{\prime}(\theta)\right]^{1 \mp p} d \theta \text { if } 0 \leqq p<\frac{\pi}{2 \operatorname{arctg} \varepsilon},
$$

so that (2.2) and (2.3) contain also estimates for the means of $\varphi^{\prime}(\theta)$. In particular, since $\pi /(2 \operatorname{arctg} \varepsilon)>1$, (2.3) is always applicable for $p=1$, and we obtain that $\varphi^{\prime}(\theta) \in L_{2}$ whenever $C$ satisfies an $\varepsilon$-condition for some $\varepsilon \geqq 0$. (For $\theta^{\prime}(\varphi) \in L_{2}$ we need $\varepsilon<1$.)

b. For $p=2$ the bounds in (2.2) and (2.3) become $\left(1-\varepsilon^{2}\right)^{-1}$ (see [8], p. 26) and $\left(1+\varepsilon^{2}\right)^{2} /\left(1-\varepsilon^{2}\right)$.

Proof. We begin with three preliminary remarks. First, we have $\left|\rho^{\prime}(\theta)\right| \rho(\theta) \mid \leqq \varepsilon$ for all $\theta$, for which $\rho^{\prime}(\theta)$ exists. For by $(0.1)$

$$
|\log \rho(\theta+h)-\log \rho(\theta)|=\left|\int_{\theta}^{\theta+h} \frac{\rho^{\prime}(t)}{\rho(t)} d t\right| \leqq \varepsilon|h|,
$$

for all $\theta$ and $h \neq 0$; this implies our proposition.

${ }^{4}$ See C. Caratheodory, Vorlesungen über reelze Funktionen, Leipzig und Berlin, 1927, pages 563 and 556. 
Next, since $C$ is rectifiable, we know by F. Riesz's theorem ([5], p. 95 ; see also [16], p. $157 \mathrm{ff}$.) that

(i) $f\left(e^{i \varphi}\right)$ is absolutely continuous, so that $\left[d f\left(e^{i \varphi}\right) / d e^{i \varphi}\right]$ exists almost everywhere and is integrable; furthermore

(ii) $f^{\prime}(z) \in H_{1}$, i.e. $\int_{0}^{2 \pi}\left|f^{\prime}\left(r e^{i \varphi}\right)\right| d \varphi \leqq A<\infty$ for all $r<1$. We claim that

$$
f^{\prime}\left(r e^{i \varphi}\right) \rightarrow \frac{d f\left(e^{i \varphi}\right)}{d e^{i \varphi}} \text { as } r \rightarrow 1 \text {, for almost all } \varphi .
$$

To prove this, let $f^{\prime}\left(r e^{i \varphi}\right) \rightarrow h\left(e^{i \varphi}\right)(r \rightarrow 1$, almost all $\varphi)$, so that by (ii) $h\left(e^{i \varphi}\right)$ is integrable and $\int_{0}^{2 \pi}\left|f^{\prime}\left(r e^{i \varphi}\right)-h\left(e^{i \varphi}\right)\right| d \varphi \rightarrow 0(r \rightarrow 1)$. Therefore, for any fixed $\varphi_{0}$,

$\left[f\left(r e^{i \varphi_{0}}\right)-f(r)\right]-r \int_{0}^{\varphi_{0}} h\left(e^{i \varphi}\right) i e^{i \varphi} d \varphi=r \int_{0}^{\varphi_{0}}\left[f^{\prime}\left(r e^{i \varphi}\right)-h\left(e^{i \varphi}\right)\right] i e^{i \varphi} d \varphi \rightarrow 0(r \rightarrow 1)$

that is

$$
f\left(e^{i \varphi_{0}}\right)=f(1)+\int_{0}^{\varphi_{0}} h\left(e^{i \varphi}\right) i e^{i \varphi} d \varphi .
$$

Differentiation yields $\left[d f\left(e^{i \varphi}\right) / d e^{2 \varphi}\right]=h\left(e^{i \varphi}\right)$ almost everywhere, which is (2.4). From now on we shall put $\left[d f\left(e^{i \varphi}\right) / d e^{i \varphi}\right]=f^{\prime}\left(e^{i \varphi}\right)$ whenever this exists.

Finally, since $f^{\prime} \in H_{1}$ and $f^{\prime} \not \equiv 0$, one knows (see, e.g., [4], p. 56) that $f^{\prime}\left(e^{i \varphi}\right)$ vanishes only on a null set.

To start the proof of theorem, let $M$ be the set of all $\varphi$ in $\langle 0,2 \pi\rangle$ for which (i) $f^{\prime}\left(e^{i \varphi}\right)$ exists and is $\neq 0$ and (ii) $\lim _{r \rightarrow 1} f^{\prime}\left(r e^{i \varphi}\right)=f^{\prime}\left(e^{i \varphi}\right)$; by our above remarks, $M$ is of measure $2 \pi$.

We consider now the function $g(z)=z f^{\prime}(z) / f(z)$, regular and $\neq 0$ in $|z|<1, g(0)=1$, and put

$$
F(z)=\log g(z)=\log |g(z)|+i \arg g(z)=u(z)+i v(z),
$$

which is regular in $|z|<1$ and vanishes at $z=0$. We study $u(z), v(z)$ foy $|z| \rightarrow 1$.

(a) Since

$$
\frac{z f^{\prime}(z)}{f(z)}=\left\{1-i \frac{\rho^{\prime}(\theta(\varphi))}{\rho(\theta(\varphi))}\right\} \theta^{\prime}(\varphi) \quad\left(z=e^{i \varphi}, \varphi \in M\right)
$$

we have $\theta^{\prime}(\varphi) \neq 0(\varphi \in M)$ and furthermore

$$
\left|g\left(r e^{i \varphi}\right)\right| \rightarrow \theta^{\prime}(\varphi)\left|1+\frac{\rho^{\prime}}{\rho} i\right|=\frac{\theta^{\prime}(\varphi)}{\cos \beta(\theta(\varphi))} \quad(r \rightarrow 1, \varphi \in M)
$$


where $\beta(\theta)$ denotes the angle between arg $w=\theta$ and the normal to $C$ at $(\rho(\theta), \theta)$. Hence

$$
u\left(r e^{i \varphi}\right) \rightarrow \log \frac{\theta^{\prime}(\varphi)}{\cos \beta(\theta(\varphi))}=u\left(e^{i \varphi}\right) \quad(r \rightarrow 1, \varphi \in M) .
$$

(b) On the other hand we have for $v(z)$

$$
\begin{aligned}
v\left(r e^{i \varphi}\right) & =\arg g\left(r e^{i \varphi}\right) \rightarrow \arg f^{\prime}\left(e^{i \varphi}\right)+\varphi-\arg f\left(e^{i \varphi}\right) \\
& =\beta(\theta(\varphi))=v\left(e^{i \varphi}\right) \quad(r \rightarrow 1, \phi \in M) .
\end{aligned}
$$

In particular, $\beta(\theta)$ exists for $\theta=\theta(\varphi), \varphi \in M$, and hence by our first remark $|\beta(\theta(\varphi))| \leqq \operatorname{arctg} \varepsilon(\varphi \in M)$.

(c) This implies that $\left|v\left(r e^{i \varphi}\right)\right| \leqq \operatorname{arctg} \varepsilon$ for $r<1$. For $v\left(r e^{i \varphi}\right)$ is harmonic in $r<1$ and clearly represents the angle between arg $w=\theta$ and the normal to the level curve corresponding to $|z|=r$, which is again starshaped. Thus $\left|v\left(r e^{i \varphi}\right)\right|<\pi / 2$, and $v\left(r e^{i \varphi}\right)$ can therefore be represented by its Poisson integral in $r<1$. Since the boundary values are $\leqq \operatorname{arctg} \varepsilon$, also $\left|v\left(r e^{i \varphi}\right)\right| \leqq \operatorname{arctg} \varepsilon(r<1)$.

For the main part of the proof, we apply a method of Zygmund ([15], p. 286). Let $p>0$ and consider

$$
1=e^{ \pm p F(0)}=\frac{1}{2 \pi i} \int_{|z|=r<1} \frac{e^{ \pm p F(z)}}{z} d z=\frac{1}{2 \pi} \int_{|z|=r<1} e^{ \pm p u(z)} \cos [p v(z)] d \varphi .
$$

By (c) and our assumption on $p$, we have $|p v(z)| \leqq p$ arctg $\varepsilon<\pi / 2$, so that the integrand in the last integral is positive for all $r<1$ and $\varphi$. Recalling (a) and (b), an application of Fatou's lemma yields

$$
\frac{1}{2 \pi} \int_{M} e^{ \pm p u\left(e^{i \varphi}\right)} \cos \left[p v\left(e^{i \varphi}\right)\right] d \varphi \leqq 1
$$

that is

$$
\frac{1}{2 \pi} \int_{M}\left[\theta^{\prime}(\varphi)\right]^{ \pm p} \frac{\cos [p \beta(\theta(\varphi))]}{[\cos \beta(\theta(\varphi))]^{ \pm p}} d \varphi \leqq 1
$$

Now we note that $|\beta(\theta(\varphi))| \leqq \operatorname{arctg} \varepsilon(\varphi \in M)$, and the fact that

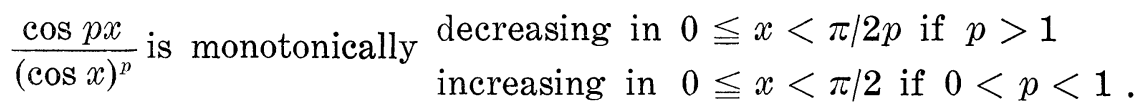

This proves our estimates (2.2) and (2.3)

We now show that our bounds are best possible. More precisely: For every $\varepsilon \geqq 0$ and for every $p$ with $0 \leqq p<\pi /(2$ arctg $\varepsilon)$, there exists a curve $C$ such that Theorem 6 holds with equality in (2.2) and (2.3), respectively.

For $\varepsilon=0$, and for $\varepsilon>0,0 \leqq p \leqq 1$ in (2.2), we simply let $C$ be 
$|w|=1, \theta^{\prime}(\varphi) \equiv 1$. For $\varepsilon>0$ and the other two cases in (2.2) and (2.3) we consider the curve $\left.C: \rho(\theta)=e^{\varepsilon ; \theta \mid}(|\theta|) \leqq \pi\right)$, which is composed of two pieces of logarithmic spirals that meet in $w=1$ and $w=-e^{\varepsilon \pi}$. Let $f(z)$ be such that $f(1)=1$ and $f(-1)=-e^{\varepsilon \pi}$.

We claim that for this mapping we have equality in (2.7) whenever $0 \leqq p<\pi /(2 \operatorname{arctg} \varepsilon)$. Since $\operatorname{tg} \beta(\theta(\varphi))= \pm \varepsilon$ for all $\varphi \neq 0$, $\pi$, this would immediately give equality in (2.2) and (2.3).

To prove equality in (2.7), we study the behaviour of $f^{\prime}(z)$ in $|z|<1$. The curve $C$ is composed of two analytic ares meeting at angles $\alpha_{1} \pi$ and $\alpha_{2} \pi$ with $\alpha_{1}=1+[2 / \pi] \operatorname{arctg} \varepsilon$ and $\alpha_{2}=1-[2 / \pi] \operatorname{arctg} \varepsilon$. By a theorem of Warschawski ([13], p. 835), we have therefore

$$
\begin{aligned}
f^{\prime}(z)(z-1)^{-(2 / \pi) \operatorname{arctg} \varepsilon} \rightarrow C_{1} \neq 0(z \rightarrow 1) \\
\text { and } f^{\prime}(z)(\dot{z}+1)^{+(2 / \pi) \operatorname{arctg} \varepsilon} \rightarrow C_{2} \neq 0(z \rightarrow-1),
\end{aligned}
$$

for unrestricted approach within $|z|<1$. Thus,

$$
f^{\prime}(z)(z+1)^{(2 / \pi) \operatorname{arctg} \varepsilon} \text { and }\left[f^{\prime}(z)\right]^{-1}(z-1)^{(2 / \pi) \operatorname{arctg} \varepsilon}
$$

are continuous in $|z| \leqq 1$, and we have for $r e^{i \varphi}, 0 \leqq r<1,0<|\varphi|<\pi$,

$$
\left|f^{\prime}\left(r e^{i \varphi}\right)\right| \leqq \frac{\text { const }}{(\pi-|\varphi|)^{(2 / \pi) \operatorname{arctg} \varepsilon}} \text { and }\left|f^{\prime}\left(r e^{i \varphi}\right)\right|^{-1} \leqq \frac{\text { const }}{|\varphi|^{(2 / \pi) \operatorname{arctg} \varepsilon}} \text {. }
$$

Therefore, if $2 p \operatorname{arctg} \varepsilon<\pi, \quad \exp \left\{ \pm p u\left(r e^{i \varphi}\right)\right\}=\left|g\left(r e^{i \varphi}\right)\right|^{ \pm p}$ is bounded by an integrable function, uniformly for all $r$ in $0 \leqq r<1$, so that Lebesgue's convergence theorem can be applied to (2.6) for $r \rightarrow 1$, giving equality in (2.7).

Finally, also the bound on $p$ is best possible. For this we simply note that by (2.5) and (2.8)

$$
\begin{aligned}
& {\left[\theta^{\prime}(\varphi)\right]^{-1}|\varphi|^{+(2 / \pi) \operatorname{arctg} \varepsilon} } \geqq D_{1}>0 \text { near } \varphi=0 \\
& \text { and } \theta^{\prime}(\varphi) \cdot(\pi-|\varphi|)^{+(2 / \pi) \operatorname{arctg} \varepsilon} \geqq D_{2}>0
\end{aligned}
$$

near $\varphi=\pi$, so that for $p=\pi /(2 \operatorname{arctg} \varepsilon)$ the functions $\left[\theta^{\prime}(\varphi)\right]^{p}$ and $\left[\theta^{\prime}(\varphi)\right]^{-p}$ are not integrable.

COROLLARY. Under the assumptions of Theorem 6, we have for $0 \leqq r<1$

$$
\frac{1}{2 \pi} \int_{0}^{2 \pi}\left|f^{\prime}\left(r e^{i \varphi}\right)\right|^{ \pm p} d \varphi \leqq \frac{\max [\rho(\theta)]^{ \pm p}}{\cos (p \operatorname{arctg} \varepsilon)} \text { if } 0 \leqq p<\frac{\pi}{2 \operatorname{arctg} \varepsilon}^{4} .
$$

For $p=\pi /(2 \operatorname{arctg} \varepsilon)$, the left-hand side need not be uniformly bounded in $0 \leqq r<1$.

${ }^{4}$ See also a similar estimate for smooth curves ([11], p. 254). 
For the proof we note that by (2.6)

$$
1=\frac{1}{2 \pi} \int_{|z|=r<1}|g(z)|^{ \pm p} \cos [p v(z)] d \varphi .
$$

Recalling $|p v(z)| \leqq p \operatorname{arctg} \varepsilon$ and that $\left.|z| f(z)\right|^{ \pm p}$ assumes its minimum for $|z|=1$, we arrive at (2.9).

II.2. An estimate for $M_{p}\left[f^{\prime}(z)-1\right]$. Theorem 6 enables us to derive an estimate for the mean of $f^{\prime}(z)-1$, which is small for small $\varepsilon$.

THEOREM 7. Let $C: \rho(\theta) e^{i \theta}$ be a Jordan curve, starshaped with respect to $w=0$, which satisfies an $\varepsilon$-condition and which lies in the ring $1 \leqq|w| \leqq 1+\varepsilon$ for some $\varepsilon \geqq 0$. Let $w=f(z)$ with $f(0)=0$, $f^{\prime}(0)>0 \operatorname{map}|z|<1$ conformally to the interior of $C$. Then we have for all $r$ with $0 \leqq r<1$

$$
\begin{aligned}
M_{p}\left[f^{\prime}\left(r e^{i \varphi}\right)-1\right] \leqq & \left\{(1+\varepsilon) \frac{\cos \operatorname{arctg} \varepsilon}{[\cos (p \operatorname{arctg} \varepsilon)]^{1 / p}}+e^{\varepsilon}\right\}\left(1+A_{p}\right) \cdot \varepsilon \\
& \text { if } 1<p<\frac{\pi}{2 \operatorname{arctg} \varepsilon},
\end{aligned}
$$

where $A_{p}$ denotes the constant in Riesz's Theorem B. The upper bound for $p$ is best possible.

This improves a theorem of Warschawski ([9], p. 566) with respect to the restrictions on $\varepsilon$ and $p$.

Proof. We first estimate $M_{p}\left[\left\{z f^{\prime}(z) / f(z)\right\}-1\right]$ (see [9], p. 565) and write by (2.5)

$$
\frac{z f^{\prime}(z)}{f(z)}-1=\left(\theta^{\prime}(\varphi)-1\right)-i \frac{\rho^{\prime}(\theta)}{\rho(\theta)} \theta^{\prime}(\varphi) \quad\left(z=e^{i \varphi}\right) .
$$

Since the left-hand side vanishes at $z=0$, Riesz's theorem gives

$$
M_{p}\left[\theta^{\prime}(\varphi)-1\right) \leqq A_{p} M_{p}\left[\frac{\rho^{\prime}(\theta(\varphi))}{\rho(\theta(\varphi))} \theta^{\prime}(\varphi)\right] \leqq A_{p} M_{p}\left[\theta^{\prime}(\varphi)\right] \cdot \varepsilon .
$$

With (2.2) and Minkowski's inequality we obtain

$$
\begin{aligned}
M_{p}\left[\frac{e^{i \varphi} f^{\prime}\left(e^{i \varphi}\right)}{f\left(e^{i \varphi}\right)}-1\right] & \leqq\left(1+A_{p}\right) M_{p}\left[\theta^{\prime}(\varphi)\right] \cdot \varepsilon \\
& \leqq\left(1+A_{p}\right) \frac{\cos \operatorname{arctg} \varepsilon}{[\cos (p \operatorname{arctg} \varepsilon)]^{1 / p}} \cdot \varepsilon .
\end{aligned}
$$

\footnotetext{
${ }^{5}$ For $0<p \leqq 1$ an estimate can be obtained by an application of Hölder's inequality.
} 
Next, we use the estimate

$$
M_{p}\left[f^{\prime}(z)-1\right] \leqq(1+\varepsilon) M_{p}\left[\frac{z f^{\prime}(z)}{f(z)}-1\right]+M_{p}\left[\frac{f(z)}{z}-1\right] \quad(|z|=r<1),
$$

where the last term is $\leqq\left(1+A_{p}\right) e^{\varepsilon} \varepsilon$; see [9], p. 564-566. Combining this with (2.11) and using the monotonicity of $M_{p}\left[\left\{z f^{\prime}(z) ! f(z)\right\}-1\right]$ with respect to $r$, we arrive at $(2.10)$.

For $p=\pi /(2 \operatorname{arctg} \varepsilon), M_{p}\left[f^{\prime}\left(r e^{i \varphi}\right)-1\right]$ need not be uniformly bounded in $0 \leqq r<1$. To see this, one has to modify our example in II.1 slightly in an obvious way so that it satisfies also $1 \leqq \rho(\theta) \leqq 1+\varepsilon$; note that only the angle $\pi \alpha_{2}$ is of importance.

\section{BIBLIOGRAPHY}

1. J. Ferrand, Sur la déformation analytique d'un domaine, C. R. Acad. Sci. Paris 221 (1945), 132-134.

2. M. S. Friberg, A new method for the effective determination of conformal maps, Thesis, University of Minnesota, 1951.

3. A. R. Marchenko, Sur la représentation conforme, C. R. (Doklady) Acad. Sci. USSR. $\mathbf{1}$ (1935), 289-290.

4. I. I. Priwalow, Randeigenschaften analytischer Funktionen, Berlin, 1956.

5. F. Riesz, Über die Randwerte einer analytischen Funktion, Math. Zeit. 18 (1923), 87-95.

6. M. Riesz, Sur les fonctions conjugées, Math. Zeit. 27 (1928), 218-244.

7. E. J. Specht, Esitmates on the mapping function and its derivatives in conformal mapping of nearly circular regions, Trans. Amer. Math. Soc., 71 (1951), 183-196.

8. S. E. Warschawski, On Theodorsen's method of conformal mapping of nearly circular regions, Quart. Applied Math. 3 (1945), 12-28.

9. _ On conformal mapping of nearly circular regions, Proc. Amer. Math. Soc., $1(1950), 562-574$.

10. $\quad$ On the degree of variation in conformal mapping of variable regions, Trans. Amer. Math. Soc., 69 (1950), 335-356.

11. , On conformal mapping of regions bounded by smooth curves, Proc. Amer. Math. Soc., 2 (1951), 254-261.

12. , On conformal mapping of variable regions, National Bureau of Standards Applied Mathematics Series, 18, (1952), 175-187.

13. On a theorem of L. Lichtenstein, Pacific J. Math., 5 (1955), 835-839.

14. On the distortion in conformal mapping of variable domains, Trans. Amer. Math. Soc. 82 (1956), 300-322.

15. A. Zygmund, Sur les fonctions conjugées, Fund. Math., 13 (1929), 284-303; Correction in $\mathbf{1 8}$ (1932), 312.

16. , Trigonometrical Series, Warsaw, 1935.

California Institute of Technology

PASADENA, CALIFORNIA AND

University of Giessen, Germany 


\section{PACIFIC JOURNAL OF MATHEMATICS}

\section{EDITORS}

Ralph S. Phillips

Stanford University

Stanford, California

M. G. Arsove

University of Washington

Seattle 5, Washington
A. L. Whiteman

University of Southern Californla

Los Angeles 7, California

Lowell J. Paige

University of California

Los Angeles 24, California

\section{ASSOCIATE EDITORS}

E. F. BECKENBACH

D. DERRY

H. L. ROYDEN

E. G. STRAUS

T. M. CHERRY

M. OHTSUKA

E. SPANIER

F. WOLF

\section{SUPPORTING INSTITUTIONS}

UNIVERSITY OF BRITISH COLUMBIA

STANFORD UNIVERSITY

CALIFORNIA INSTITUTE OF TECHNOLOGY

UNIVERSITY OF CALIFORNIA

MONTANA STATE UNIVERSITY

UNIVERSITY OF TOKYO

UNIVERSITY OF UTAH

UNIVERSITY OF NEVADA

NEW MEXICO STATE UNIVERSITY

OREGON STATE UNIVERSITY

UNIVERSITY OF OREGON

OSAKA UNIVERSITY

WASHINGTON STATE UNIVERSITY

UNIVERSITY OF WASHINGTON

UNIVERSITY OF SOUTHERN CALIFORNIA

AMERICAN MATHEMATICAL SOCIETY CALIFORNIA RESEARCH CORPORATION SPACE TECHNOLOGY LABORATORIES NAVAL ORDNANCE TEST STATION 


\section{Pacific Journal of Mathematics}

\section{Vol. 12, No. $1 \quad$ January, 1962}

Jonathan L. Alperin, Groups with finitely many automorphisms $\ldots \ldots \ldots \ldots \ldots \ldots \ldots \ldots$

Martin Arthur Arkowitz, The generalized Whitehead product ................ 7

John D. Baum, Instability and asymptoticity in toplogical dynamics . . . . . . . . . . 25

William Aaron Beyer, Hausdorff dimension of level sets of some Rademacher series .... $\quad 35$

Frank Herbert Brownell, III, A note on Cook's wave-matrix theorem . . . . . . . . . . . . . 47

Gulbank D. Chakerian, An inequality for closed space curves ................. 53

Inge Futtrup Christensen, Some further extensions of a theorem of Marcinkiewicz ....... 59

Charles Vernon Coffman, Linear differential equations on cones in Banach spaces . . . . . 69

Eckford Cohen, Arithmetical notes. III. Certain equally distributed sets of integers . . . . . 77

John Irving Derr and Angus E. Taylor, Operators of meromorphic type with multiple poles

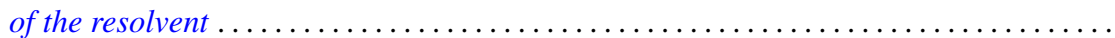

Jacob Feldman, On measurability of stochastic processes in products space .............

Robert S. Freeman, Closed extensions of the Laplace operator determined by a general class of boundary conditions, for unbounded regions ......................

Robert E. Fullerton, Geometric structure of absolute basis systems in a linear topological

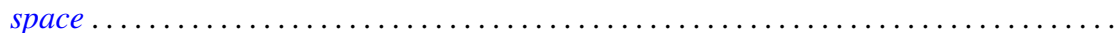

Dieter Gaier, On conformal mapping of nearly circular regions

Andrew Mattei Gleason and Hassler Whitney, The extension of linear functionals defined

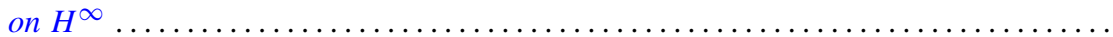

Seymour Goldberg, Closed linear operators and associated continuous linear

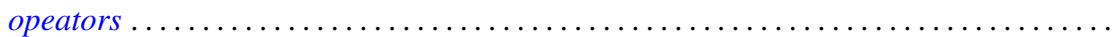

Basil Gordon, Aviezri Siegmund Fraenkel and Ernst Gabor Straus, On the determination of sets by the sets of sums of a certain order

Branko Grünbaum, The dimension of intersections of convex sets. .

Paul Daniel Hill, On the number of pure subgroups

Robert Peter Holten, Generalized Goursat problem . .

Alfred Horn, Eigenvalues of sums of Hermitian matrices ...........

Henry C. Howard, Oscillation and nonoscillation criteria for

$$
y^{\prime \prime}(x)+f(y(x)) p(x)=0
$$

Taqdir Husain, $S$-spaces and the open mapping theorem ...

Richard Eugene Isaac, Markov processes and unique stationary probability measures ...

John Rolfe Isbell, Supercomplete spaces ....................

John Rolfe Isbell, On finite-dimensional uniform spaces. II .........

N. Jacobson, A note on automorphisms of Lie algebras ..............

Antoni A. Kosinski, A theorem on families of acyclic sets and its applications

Marvin David Marcus and H. Minc, The invariance of symmetric functions of singular values...

Ralph David McWilliams, A note on weak sequential convergence.

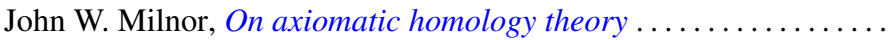

Victor Julius Mizel and Malempati Madhusudana Rao, Nonsymmetric projections in

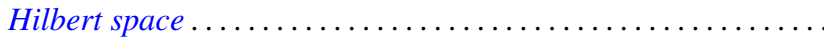

Calvin Cooper Moore, On the Frobenius reciprocity theorem for locally compact

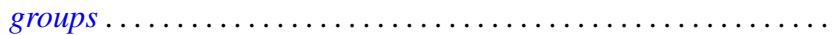

Donald J. Newman, The Gibbs phenomenon for Hausdorff means . 